\title{
Alkylresorcinol metabolites in urine correlate with the intake of whole grains and cereal fibre in free-living Swedish adults
}

\author{
Matti Marklund ${ }^{1 *}$, Rikard Landberg ${ }^{1}$, Agneta Andersson ${ }^{2}$, Per Åman ${ }^{1}$ and Afaf Kamal-Eldin ${ }^{1,3}$ \\ ${ }^{1}$ Department of Food Science, Swedish University of Agricultural Sciences, Box 7051, SE-750 07 Uppsala, Sweden \\ ${ }^{2}$ Department of Food, Nutrition and Dietetics, Uppsala University, Box 560, SE-751 22 Uppsala, Sweden \\ ${ }^{3}$ Department of Food Science, Faculty of Food and Agriculture, United Arab Emirates University (UAEU), Box 17551, \\ Al Ain, UAE \\ (Submitted 6 September 2011 - Final revision received 16 January 2012 - Accepted 27 January 2012 - First published online 3 April 2012)
}

\section{Abstract}

Alkylresorcinols (AR) have been established as short/medium-term biomarkers for whole grain (WG) wheat and rye intake; and AR metabolites, 3,5-dihydroxybenzoic acid and 3-(3,5-dihydroxyphenyl)-propanoic acid, have been suggested as complementary biomarkers to AR. The present study examined the medium-term reproducibility and relative validity of urinary AR metabolites as biomarkers for WG and cereal fibre intake. A total of sixty-six free-living Swedes completed $3 \mathrm{~d}$ weighed food records and provided single $24 \mathrm{~h}$ urine collections and morning urine spot samples on two occasions, 2-3 months apart. The medium-term reproducibility of urinary AR metabolites was moderate when assessed in $24 \mathrm{~h}$ collections and lower in creatinine (CR)-adjusted morning urine. Mean AR metabolite $24 \mathrm{~h}$ excretions correlated well with total WG $\left(r_{\mathrm{s}} 0.31-0.52, P<0.05\right)$ and cereal fibre $\left(r_{\mathrm{s}} 0.46-0.58, P<0.001\right)$ intake on both occasions. As expected, correlations with WG $\left(r_{\mathrm{s}} 0.28-0.38, P<0.05\right)$ and cereal fibre $\left(r_{\mathrm{s}} 0.35-0.42, P<0 \cdot 01\right)$ were weaker for mean CR-adjusted AR metabolite concentrations in spot samples of morning urine, although the adjusted concentrations correlated well with $24 \mathrm{~h}$ urinary excretion $\left(r_{\mathrm{s}} 0.69-0.73, P<0.001\right)$. Adjustment for intra-individual variations substantially improved the correlations between intake and excretion. These findings suggest that urinary AR metabolites can successfully reflect the medium-term intake of WG and cereal fibre when adjusted for intra-individual variation in this population, where rye was the major contributor to high WG intake. The performance of urinary AR metabolites as medium-term biomarkers appears to be comparable to that of fasting plasma AR concentration in this population.

\section{Key words: Biomarkers: Whole grains: Validity: Reproducibility}

Alkylresorcinols (AR) are biomarkers for the intake of whole grain (WG) wheat and rye that can be used to assess compliance in intervention studies ${ }^{(1-4)}$ and they may be useful in prospective cohort studies ${ }^{(5)}$. Cereal AR are phenolic lipids found, as several homologues, in the bran of rye and wheat $^{(6)}$ and are therefore present in WG wheat and rye products, but absent or found in very low concentrations in refined products of the same cereals and in other foods ${ }^{(7)}$. Although barley contains small amounts, the contribution of this cereal to AR intake is minute due to the low amounts of WG barley generally consumed by humans ${ }^{(8)}$. Approximately $60 \%$ of ingested AR are absorbed in the small intestine. AR disappear rather quickly from plasma ( $t_{\frac{1}{2}}$ about $5 \mathrm{~h}$ ), and hence plasma AR reflect short/medium-term intake ${ }^{(9)}$. The metabolism of AR is thought to be similar to that of tocopherols, which is initiated by cytochrome $\mathrm{P} 450$-mediated $\omega$-oxidation, followed by several cycles of $\beta$-oxidation leading to the formation of more hydrophilic end products ${ }^{(10)}$. The major AR metabolites are two phenolic acids, 3,5-dihydroxybenzoic acid (DHBA) and 3-(3,5-dihydroxyphenyl)-propanoic acid (DHPPA), which are excreted in urine ${ }^{(11)}$. In addition to the aforementioned oxidative metabolism, AR and/or their metabolites are subjected to conjugation reactions and conjugated AR metabolites have been identified in plasma ${ }^{(12)}$ and urine $^{(13,14)}$. Compared with intact $\mathrm{AR}$, their metabolites appear to have a longer residence time in plasma and thus AR metabolites could act as longer-term biomarkers ${ }^{(15,16)}$. So far, reproducibility (i.e. stability of concentration or excretion over a period of time) of urinary AR metabolites has not been assessed in any population. In a controlled bran and WG intervention study, urinary excretion of AR metabolites increased with elevated AR intake, although the proportion

Abbreviations: AR, alkylresorcinols; DHBA, 3,5-dihydroxybenzoic acid; DHPPA, 3-(3,5-dihydroxyphenyl)-propanoic acid; CR, creatinine; ICC, intra-class correlation coefficient; WG, whole grains.

*Corresponding author: M. Marklund, fax +46186729 95, email matti.marklund@slu.se 
of ingested AR recovered in urine as DHBA and DHPPA decreased as intake increased ${ }^{(3)}$. Excretion of urinary $A R$ metabolites has previously been shown to correlate with the intake of rye ${ }^{(17)}$ and cereal fibre $e^{(18)}$ in free-living Finnish women. In addition, urinary excretion of DHPPA was associated with the intake of WG wheat and rye in free-living American adults ${ }^{(19)}$. Previous analyses of urinary AR metabolites have been performed on $12 \mathrm{~h}$ to $72 \mathrm{~h}$ urine collections ${ }^{(3,14,17,19-21)}$. Although urine collections covering several micturitions are likely to represent more complete biomarker excretion, these collections are seldom available in large epidemiological studies ${ }^{(22)}$. Instead, morning urine or spot samples adjusted for diuresis are commonly used for urine analysis of exposure biomarkers ${ }^{(23)}$.

The present study evaluated urinary AR metabolites as biomarkers by estimating the medium-term (2-3 months) reproducibility and their relative validity compared with self-reported intake of WG, cereal fibre and AR in free-living Swedish women and men. These evaluations were conducted for AR metabolite excretion in $24 \mathrm{~h}$ collections and morning urine AR metabolite concentration adjusted for creatinine (CR).

\section{Materials and methods}

\section{Participants and study design}

Participant recruitment and study design have been thoroughly described previously ${ }^{(24)}$. In brief, we initially recruited ninety-one participants for the study and instructed them to adhere to their habitual diet and to complete $3 \mathrm{~d}$ weighed food records on two occasions, approximately 2-3 months apart. A total of thirteen participants withdrew from the study for personal reasons or provided incomplete food records; and six participants with food intake level (daily energy intake divided by $\mathrm{BMR}) \leq 1.08$ were excluded due to underreported energy intake as previously described ${ }^{(24)}$. On each occasion, the participants provided a spot sample of morning urine (approximately $25 \mathrm{ml}$ ) from the final day of the $3 \mathrm{~d}$ weighed food records and a $24 \mathrm{~h}$ urine collection (except the $25 \mathrm{ml}$ morning urine) from the same day. To prevent microbial growth, the participants added $9 \mathrm{ml} 20 \% \mathrm{HCl}$ to each $24 \mathrm{~h}$ collection. We recorded the volumes of $24 \mathrm{~h}$ urine collections and morning urine samples, kept approximately $50 \mathrm{ml}$ of the $24 \mathrm{~h}$ urine collections for analysis and stored the samples at $-80^{\circ} \mathrm{C}$ until analysis. Besides, six participants provided incomplete $24 \mathrm{~h}$ urine collection or morning urine samples, leaving in total sixty-six participants (fifty females, sixteen males) with satisfactory data for inclusion in the present study. We measured body weight and height, calculated BMI and recorded the age of the participants on the second occasion. The mean age and BMI of the participants were 44 (SD 17) years and $24(\mathrm{SD} 4) \mathrm{kg} / \mathrm{m}^{2}$, respectively. This study was conducted according to the guidelines laid down in the Declaration of Helsinki and all procedures involving human subjects were approved by the Regional Ethical Review Board in Uppsala (log. no. 2008:040). Written informed consent was obtained from all subjects.

\section{Intake estimation}

Assessments of self-recorded dietary intake and analysis of AR in foods were made as described previously ${ }^{(24)}$. In brief, we calculated nutrient intake using the food database of the Swedish National Food Administration (PC-kost 2008-03-06) and a computerised calculation program (Diet XP; kost och Näringsdata $\mathrm{AB}$ ). We defined WG in accordance with the definition of the American Association of Cereal Chemists ${ }^{(25)}$ and estimated the content based on product declarations of reported products. We compiled the content of WG as $\mathrm{g}$ $\mathrm{WG} / 100 \mathrm{~g}$ of ready-to-eat product in reported products to a WG database. After AR quantification in reported foods by $\mathrm{GC}^{(26)}$, we added the content to the WG database.

\section{Urine analysis}

We determined the urinary concentrations of AR metabolites, DHBA and DHPPA, by an in-house validated GC-MS method described previously ${ }^{(14,21)}$. In brief, we mixed $50 \mu \mathrm{l}$ urine with internal standard $(15 \mu \mathrm{mol}$ syringic acid in $10 \mu \mathrm{l}$ methanol) and $740 \mu \mathrm{l}$ hydrolysis solution $(0 \cdot 1 \mathrm{M}$-sodium acetate buffer ( $\mathrm{pH} 5 \cdot 0$ ) containing both $\beta$-glucuronidase and sulphatase activity). After incubation overnight at $37^{\circ} \mathrm{C}$ and addition of $15 \mu \mathrm{l}$ concentrated $\mathrm{HCl}$, we proceeded with ethyl acetate extraction $(2 \times 3 \mathrm{ml})$. As the next step, we performed solid phase extraction on Oasis ${ }^{\circledR}$ MAX cartridges and eluted the AR metabolites with $3 \mathrm{ml} 2 \%$ formic acid in methanol. Finally, we evaporated $2 \mathrm{ml}$ of the eluate to complete dryness, derivatised by incubation with $100 \mu \mathrm{l} 1 \%$ trimethylchlorosilane in $N, O$-bis-(trimethylsilyl)trifluoroacetamide, and analysed the samples by GC-MS in selected ion recording mode. We analysed all samples as single samples and included triplicates of two control samples in each batch. Within- and between-batch $\mathrm{CV}$ of both metabolites was $<8 \%$ and $\leq 11 \%$, respectively. We calculated $24 \mathrm{~h}$ excretion by multiplying the AR metabolite concentration by the recorded volume of $24 \mathrm{~h}$ urine collections. We analysed CR concentration enzymatically in morning urine samples with an Architect ci8200 (Abbott). Concentrations of AR metabolites in morning urine are reported as $\mathrm{mmol} / \mathrm{mol} \mathrm{CR}$.

\section{Statistical analysis}

The data are expressed as means and standard deviations, unless otherwise stated. We log-transformed variables with non-normal distribution $(P<0 \cdot 05$, Shapiro-Wilk test) before further analysis and evaluated differences in intake and excretion between occasions by paired $t$ tests. Furthermore, we calculated inter- and intra-individual $\mathrm{CV}$ of intakes and excretions by using random effect models for the estimation of variance components. To evaluate the stability of intake and urinary excretion of AR metabolites, we assessed the medium-term reproducibility over a 2-3-month period by calculating the intra-class correlation coefficient (ICC). ICC can be defined as the proportion of the total variation that is due to variation between participants. A high ICC indicates a low variation between measurements in the same participant 
in relation to the variation between participants, and thus the reproducibility between measurements is good. We calculated ICC values and their $95 \%$ CI as described previously ${ }^{(24)}$. In order to address the relative validity of urinary AR metabolites as biomarkers, we calculated Spearman's rank $r_{\mathrm{s}}$ between dietary intake and urinary excretion of AR metabolites, on the same and opposite occasions and for means of occasions. We used a freely available SAS-macro ${ }^{(27)}$ to adjust observed correlations for intra-individual variation in intake and excretion separately, and calculated 95\% CI of adjusted $r_{\mathrm{s}}$ according to Rosner \& Glynn ${ }^{(28)}$. To calculate metabolite recovery, we divided the mean $\mathrm{AR}$ metabolite excretion ( $\mu \mathrm{mol})$ from the two occasions by the mean daily AR intake ( $\mu \mathrm{mol})$ estimated from two $3 \mathrm{~d}$ weighed food records. Furthermore, we divided the participants consuming AR on both occasions ( $n$ 62) into quartiles based on their mean AR intake and calculated $P$ for trend for recovery, using a general linear model, with the log-transformed metabolite recovery as dependent variable and quartile median AR intake as independent variable. We performed all statistical analyses using SAS 9.1 (SAS Institute, Inc.) and considered $P<0.05$ statistically significant.

\section{Results}

\section{Intake of whole grain, alkylresorcinols and cereal fibre}

The intake of macronutrients did not differ between the two occasions and the 2-3-month reproducibility of the $3 \mathrm{~d}$ weighed food records was high for energy, carbohydrate, protein and dietary fibre, but lower for fat and cereal fibre (Table 1). Similarly, the intake of WG and AR did not differ between the occasions (Table 1). The mean intake of WG was about $70 \mathrm{~g}$ and the major source of WG was rye, followed by wheat and oats (Table 1 ). The $2-3$-month reproducibility of intakes of total WG, WG rye and WG oats was similar to that of cereal fibre, but the reproducibility of WG wheat intake was lower (Table 1). The mean daily AR intake was about $35 \mathrm{mg}$ (Table 1). Reproducibility of total AR intake over 2-3 months was similar to that of WG intake (Table 1).

\section{Urinary excretion of alkylresorcinol metabolites and reproducibility}

Excretion of AR metabolites did not differ significantly between the two occasions (Table 2). DHPPA was excreted to a greater extent than DHBA and the median DHPPA:DHBA ratio was 1.7 (interquartile range: 0.90 ). The $24 \mathrm{~h}$ excretion of AR metabolites over 2-3 months was moderately stable as indicated by ICC and the reproducibility was slightly higher for DHPPA than for DHBA (Table 2). The median DHPPA:DHBA ratio in morning urine was similar to the ratio in $24 \mathrm{~h}$ urine collections and CR-adjusted concentrations did not differ significantly between occasions (Table 2). The reproducibility of metabolite concentration in morning urine over 2-3 months was lower than for $24 \mathrm{~h}$ excretion of the same metabolites (Table 2). The $24 \mathrm{~h}$ urine excretion correlated

Table 1. Reported intakes of macronutrients, whole grains (WG) and alkylresorcinols (AR) among free-living Swedish adults on two separate occasions $2-3$ months apart and the medium-term reproducibility ${ }^{\star} \dagger$

(Mean values and standard deviations, $n 66$ )

\begin{tabular}{|c|c|c|c|c|c|c|c|c|c|}
\hline & \multicolumn{2}{|c|}{$\begin{array}{c}\text { First } \\
\text { occasion } \neq\end{array}$} & \multicolumn{2}{|c|}{$\begin{array}{c}\text { Second } \\
\text { occasion }\end{array}$} & \multirow[b]{2}{*}{$\mathrm{CV}_{\text {Inter }}(\%) \S$} & \multirow[b]{2}{*}{$\mathrm{CV}_{\text {Intra }}(\%) \S$} & \multirow[b]{2}{*}{ ICC $\|$} & \multirow[b]{2}{*}{$95 \% \mathrm{Cl}$} & \multirow[b]{2}{*}{$n$} \\
\hline & Mean & SD & Mean & SD & & & & & \\
\hline \multicolumn{10}{|l|}{ Macronutrients } \\
\hline Energy (MJ/d) & $9 \cdot 2$ & $2 \cdot 0$ & 9.4 & 1.8 & 17 & 11 & 0.71 & $0.57,0.81$ & 66 \\
\hline Carbohydrates (g/d) & 261 & 77 & 261 & 66 & 23 & 14 & 0.74 & $0.61,0.83$ & 66 \\
\hline Protein (g/d) & 87 & 24 & 85 & 23 & 24 & 13 & 0.77 & $0.66,0.85$ & 66 \\
\hline Fat $(g / d)$ & 79 & 23 & 84 & 25 & 21 & 22 & 0.47 & $0.30,0.65$ & 66 \\
\hline Dietary fibre $(\mathrm{g} / \mathrm{d})$ & 29 & 15 & 29 & 12 & 38 & 19 & 0.79 & $0.69,0.87$ & 66 \\
\hline Cereal fibre $(\mathrm{g} / \mathrm{d})$ & 13 & 9 & 11 & 8 & 46 & 51 & 0.45 & $0.27,0.64$ & 64 \\
\hline \multicolumn{10}{|l|}{ WG $(g / d)$} \\
\hline Total & 70 & 58 & 69 & 46 & 50 & 54 & 0.45 & $0.27,0.65$ & 64 \\
\hline Rye & 29 & 27 & 28 & 30 & 64 & 69 & 0.46 & $0.28,0.65$ & 60 \\
\hline Wheat & 19 & 22 & 15 & 16 & 58 & 92 & 0.28 & $0.10,0.65$ & 47 \\
\hline Rye + wheat & 47 & 35 & 43 & 33 & 45 & 61 & 0.35 & $0.17,0.58$ & 63 \\
\hline Oats & 19 & 20 & 19 & 24 & 69 & 65 & 0.53 & $0.33,0.71$ & 48 \\
\hline Rice & 2 & 9 & 2 & 9 & $-{ }^{\star \star}$ & $-{ }^{* *}$ & $-^{\star \star}$ & - & 1 \\
\hline Barley & 2 & 7 & 4 & 9 & 22 & 92 & 0.05 & $0.00,1.00$ & 13 \\
\hline Maize & 0 & & $2^{* *}$ & 6 & $-\star \star *$ & $-{ }^{\star *}$ & - & - & 0 \\
\hline \multicolumn{10}{|l|}{$\operatorname{AR}(\mathrm{mg} / \mathrm{d})$} \\
\hline Total & 34 & 27 & 33 & 29 & 55 & 56 & 0.48 & $0.29,0.69$ & 62 \\
\hline
\end{tabular}

ICC, intra-class correlation coefficient.

* Estimated by $3 \mathrm{~d}$ weighed food records.

† Reproducibility assessed by ICC.

$\ddagger$ No significant differences between occasions were observed (paired $t$ test on log-transformed data).

$\S$ Variance components (inter- and intra-individual variance) expressed as CV (\%) were estimated on log-transformed values by a random-effect model.

\| Defined as inter-individual variance/total variance.

I Number of participants with intake on both occasions, and thus included in the calculations of CV and ICC.

${ }^{\star \star}$ Too few participants $(n<10)$ with reported intakes $>0 \mathrm{~g} / \mathrm{d}$ on both occasions. 
Table 2. Daily excretion of alkylresorcinol (AR) metabolites measured 2-3 months apart and their medium-term reproducibility in free-living Swedish adults* $^{*}$

(Geometric means and $95 \%$ confidence intervals, $n$ 66)

\begin{tabular}{|c|c|c|c|c|c|c|c|c|}
\hline \multirow[b]{2}{*}{ AR metabolite } & \multicolumn{2}{|c|}{ First occasion† } & \multicolumn{2}{|c|}{ Second occasion† } & \multirow[b]{2}{*}{$\mathrm{CV}_{\text {Inter }}(\%) \ddagger$} & \multirow[b]{2}{*}{$\mathrm{CV}_{\text {Intra }}(\%) \ddagger$} & \multirow[b]{2}{*}{ ICC§ } & \multirow[b]{2}{*}{$95 \% \mathrm{Cl}$} \\
\hline & Mean & $95 \% \mathrm{Cl}$ & Mean & $95 \% \mathrm{Cl}$ & & & & \\
\hline \multicolumn{9}{|l|}{ DHBA } \\
\hline $24 \mathrm{~h}$ Urine $(\mu \mathrm{mol} / \mathrm{d})$ & 17 & 14,20 & 17 & 15,21 & 50 & 54 & 0.46 & $0.28,0.65$ \\
\hline Morning spot urine $(\mathrm{mmol} / \mathrm{mol} \mathrm{CR})$ & 1.3 & $1.0,1.6$ & 1.4 & $1 \cdot 2,1 \cdot 7$ & 47 & 67 & 0.32 & $0.15,0.56$ \\
\hline \multicolumn{9}{|l|}{ DHPPA } \\
\hline $24 \mathrm{~h}$ Urine $(\mu \mathrm{mol} / \mathrm{d})$ & 28 & 23,33 & 29 & 24,34 & 52 & 50 & 0.51 & $0.34,0.68$ \\
\hline Morning spot urine $(\mathrm{mmol} / \mathrm{mol} \mathrm{CR})$ & $2 \cdot 4$ & $1 \cdot 9,2.9$ & 2.5 & $2 \cdot 1,3 \cdot 0$ & 50 & 64 & 0.37 & $0.19,0.59$ \\
\hline \multicolumn{9}{|l|}{ Total } \\
\hline $24 \mathrm{~h}$ Urine $(\mu \mathrm{mol} / \mathrm{d})$ & 45 & 38,54 & 47 & 40,55 & 49 & 50 & 0.48 & $0.30,0.66$ \\
\hline Morning spot urine $(\mathrm{mmol} / \mathrm{mol} \mathrm{CR})$ & 3.7 & $3 \cdot 0,4 \cdot 6$ & $4 \cdot 0$ & $3 \cdot 4,4 \cdot 7$ & 47 & 62 & 0.36 & $0.19,0.58$ \\
\hline
\end{tabular}

ICC, intra-class correlation coefficient; DHBA, 3,5-dihydroxybenzoic acid; CR, creatinine; DHPPA, 3-(3,5-dihydroxyphenyl)-propanoic acid.

${ }^{*}$ Reproducibility assessed by ICC.

† No significant differences between occasions were observed (paired $t$ test on log-transformed data).

$\ddagger$ Variance components (inter- and intra-individual variance) expressed as CV (\%) were estimated on log-transformed values by a random-effect model.

$\S$ Defined as inter-individual variance/total variance.

well with the same day's morning urine CR-adjusted concentration of DHBA $\left(r_{\mathrm{s}} \quad 0.69-0.70, \quad P<0.001\right)$ and DHPPA $\left(r_{\mathrm{s}} 0 \cdot 70-0 \cdot 72, P<0 \cdot 001\right)$ on both occasions (data not shown).

Urinary excretion of alkylresorcinol metabolites in relation to intake of alkylresorcinol, whole grain and fibre

Self-reported intakes of WG rye and wheat, WG rye and cereal fibre were significantly correlated with $24 \mathrm{~h}$ urinary AR metabolite excretion, regardless of whether intake and excretion were measured on the same occasion or 2-3 months apart (Table 3). Urine concentrations adjusted for CR in morning spot samples were correlated with the same intake parameters if intake and urine concentration were measured on the same occasion (Table 4). Generally, intake of total WG was correlated with urinary AR metabolites ( $24 \mathrm{~h}$ excretion and morning urine) when measured on the same occasion. Intake of WG wheat was only correlated with excretion of DHBA $\left(r_{\mathrm{s}} 0.36, P<0.01\right)$ and total AR metabolites $\left(r_{\mathrm{s}} 0 \cdot 28, P<0.05\right)$ on the first occasion (Table 4). As expected, urinary AR metabolite excretion $(24 \mathrm{~h}$ collections) and concentrations (morning spot samples) were not correlated with intakes of other cereals consumed as WG (oats, barley, maize and rice). Intake of total fibre was correlated with $24 \mathrm{~h}$ urinary excretion of AR metabolites, when intake and excretion were measured on the same occasion $\left(r_{s} 0 \cdot 36-0.53\right.$, $P<0 \cdot 01)$ and when measured $2-3$ months apart $\left(r_{\mathrm{s}} 0 \cdot 27-0 \cdot 51\right.$, $P<0.05)$. The CR-adjusted concentrations of AR metabolites in morning urine were significantly correlated with intake of total fibre only if intake and urine concentration were measured on the same occasion $\left(r_{\mathrm{s}} 0.33-0 \cdot 40, P<0.05\right)$. Intakes of AR (total and individual homologues) were significantly $(P<0 \cdot 01)$ correlated with AR metabolite $24 \mathrm{~h}$ urinary excretion (irrespective of occasion) and adjusted concentration in morning urine spot samples (at the same occasion) (data not shown). Mean AR metabolite excretions ( $24 \mathrm{~h}$ and morning urine) of two occasions were correlated with intakes of total WG, WG rye and wheat, WG rye, total fibre and cereal fibre at both occasions and their means (Tables 3 and 4). As expected, the correlations became stronger after adjustment for intra-individual variation in intake (Tables 3 and 4). Correlations between intake and morning urine concentration became stronger after adjustment for intra-individual variation in biomarker compared to after adjustment for intake variation (data not shown). For correlations between intake and $24 \mathrm{~h}$ excretion, adjusting for biomarker and intake gave similar correlation coefficients. Recovery of ingested AR excreted as metabolites in urine was lower in participants with high AR intake than in subjects consuming lower amounts of AR (Fig. 1).

\section{Discussion}

In a previous study, we showed that fasting plasma AR concentration was moderately stable over $2-3$ months in freeliving Swedish participants and correlated relatively well with the combined intake of WG rye and wheat ${ }^{(24)}$. In the present study, the reproducibility and relative validity of urinary AR metabolites as biomarkers for WG intake were assessed in the same population.

Urine is commonly used to assess exposure biomarkers ${ }^{(29)}$ and is an advantageous site of biomarker measurement due to ease of collection, with little interference to the participant's daily life. Compared with plasma, which only indicates biomarker status at a certain time-point, urine provides a cumulative biomarker measurement. Some urinary biomarkers are recovery biomarkers that quantitatively reflect intake (e.g. $24 \mathrm{~h}$ urinary nitrogen as a biomarker for protein intake) and can be compared with traditional dietary assessment methods on the same scale ${ }^{(30)}$. Although $24 \mathrm{~h}$ urine collections represent the complete daily excretion of the biomarker, they are subjected to sampling complications and are seldom available in large epidemiological studies due to complex logistics ${ }^{(22)}$. An alternative is spot urine samples, which involve minimal participant inconvenience, are simple to collect and are relatively easy to obtain in large population studies ${ }^{(23,31)}$. The concentrations in spot samples are subjected to fluctuations due to factors unrelated to exposure, but these variations can be minimised by adjustments to diuresis, e.g. $\mathrm{CR}^{(23,29)}$. 
Table 3. Observed and adjusted Spearman's rank $r_{\mathrm{s}}$ between $24 \mathrm{~h}$ urinary excretion of alkylresorcinol (AR) metabolites and intake of total whole grain (WG), WG rye, WG wheat and cereal fibre†‡

(Mean values with their $95 \%$ confidence intervals)

\begin{tabular}{|c|c|c|c|c|c|c|c|}
\hline & & \multicolumn{2}{|c|}{ DHBA } & \multicolumn{2}{|c|}{ DHPPA } & \multicolumn{2}{|c|}{ DHBA + DHPPA } \\
\hline & & First occasion & Mean & First occasion & Mean & First occasion & Mean \\
\hline \multicolumn{8}{|l|}{ Total WG } \\
\hline First occasion & $r_{\mathrm{s}}$ & $0.55^{\star \star \star}$ & $0.52^{\star \star \star}$ & $0.49^{\star \star \star}$ & $0.48^{\star \star *}$ & $0.50^{\star \star \star}$ & $0.49^{\star \star \star}$ \\
\hline Second occasion & $r_{\mathrm{s}}$ & $0.26^{\star}$ & $0.43^{\star \star \star}$ & 0.21 & $0.31^{*}$ & 0.22 & $0.35^{\star \star}$ \\
\hline Mean & $r_{\mathrm{s}}$ & $0.46^{\star \star \star}$ & $0.54^{\star \star \star}$ & $0.40^{\star * *}$ & $0.44^{\star \star \star}$ & $0.41^{\star \star \star}$ & $0.47^{\star * \star}$ \\
\hline Adjusted mean§ & $r_{\mathrm{s}}$ & 0.54 & 0.69 & 0.48 & 0.60 & 0.48 & 0.63 \\
\hline & $95 \% \mathrm{Cl}$ & $0.29,0.72$ & $0.47,0.83$ & $0.22,0.68$ & $0.36,0.77$ & $0.25,0.70$ & $0.40,0.79$ \\
\hline \multicolumn{8}{|l|}{ WG rye and wheat } \\
\hline First occasion & $r_{\mathrm{s}}$ & $0.61^{\star \star \star}$ & $0.60^{\star \star \star}$ & $0.57^{\star \star \star}$ & $0.58^{\star \star *}$ & $0.59^{\star \star \star}$ & $0.59^{\star \star \star}$ \\
\hline Second occasion & $r_{\mathrm{s}}$ & $0.33^{\star \star}$ & $0.55^{\star \star \star}$ & $0.28^{*}$ & $0.41^{\star \star \star}$ & $0.29^{*}$ & $0.46^{\star * \star}$ \\
\hline Mean & $r_{\mathrm{s}}$ & $0.54^{\star \star *}$ & $0.67^{\star \star \star}$ & $0.49^{* * *}$ & $0.57^{\star \star \star}$ & $0.51^{* * *}$ & $0 \cdot 61^{\star * *}$ \\
\hline Adjusted mean & $r_{\mathrm{s}}$ & 0.64 & 0.83 & 0.61 & 0.75 & 0.64 & 0.79 \\
\hline & $95 \% \mathrm{Cl}$ & $0.40,0.80$ & $0.66,0.92$ & $0.36,0.78$ & $0.54,0.87$ & $0.40,0.80$ & $0.59,0.89$ \\
\hline \multicolumn{8}{|l|}{ WG rye } \\
\hline First occasion & $r_{\mathrm{s}}$ & $0.56^{\star \star \star}$ & $0.67^{\star \star \star}$ & $0.61^{\star \star \star}$ & $0.67^{\star \star \star}$ & $0.62^{\star \star \star}$ & $0.70^{\star \star \star}$ \\
\hline Second occasion & $r_{\mathrm{s}}$ & $0 \cdot 33^{\star \star}$ & $0 \cdot 56^{\star \star \star}$ & $0 \cdot 36^{\star \star}$ & $0.55^{\star \star \star}$ & $0 \cdot 35^{\star \star}$ & $0 \cdot 56^{\star \star \star}$ \\
\hline Mean & $r_{\mathrm{s}}$ & $0.50^{\star \star *}$ & $0.70^{\star \star \star}$ & $0.54^{\star * \star}$ & $0.68^{* \star *}$ & $0.54^{\star \star *}$ & $0.70^{\star \star \star}$ \\
\hline Adjusted mean & $r_{\mathrm{s}}$ & 0.56 & 0.83 & 0.65 & 0.84 & 0.65 & 0.86 \\
\hline & $95 \% \mathrm{Cl}$ & $0.32,0.73$ & $0.67,0.92$ & $0.43,0.80$ & $0.68,0.92$ & $0.44,0.80$ & $0.72,0.94$ \\
\hline \multicolumn{8}{|l|}{ WG wheat } \\
\hline First occasion & $r_{\mathrm{s}}$ & $0.36^{\star \star}$ & 0.23 & 0.24 & 0.15 & $0.27^{\star}$ & 0.16 \\
\hline Second occasion & $r_{\mathrm{s}}$ & 0.11 & 0.11 & -0.04 & -0.03 & -0.02 & -0.01 \\
\hline Mean & $r_{\mathrm{s}}$ & $0 \cdot 28^{*}$ & $0 \cdot 19$ & 0.14 & 0.08 & 0.17 & 0.09 \\
\hline Adjusted mean & $r_{\mathrm{s}}$ & 0.33 & 0.22 & 0.15 & $0 \cdot 10$ & 0.18 & 0.12 \\
\hline & $95 \% \mathrm{Cl}$ & $0.04,0.57$ & $-0.07,0.48$ & $-0.14,0.41$ & $-0.19,0.37$ & $-0.11,0.45$ & $-0.17,0.39$ \\
\hline \multicolumn{8}{|l|}{ Cereal fibre } \\
\hline First occasion & $r_{\mathrm{s}}$ & $0.60^{\star \star \star}$ & $0.58^{\star \star \star}$ & $0.56^{\star \star \star}$ & $0.56^{\star \star \star}$ & $0.57^{\star \star \star}$ & $0.57^{\star \star \star}$ \\
\hline Second occasion & $r_{\mathrm{s}}$ & $0.33^{\star *}$ & $0.50^{\star \star *}$ & $0.36^{* *}$ & $0.46^{\star \star *}$ & $0.34^{\star *}$ & $0.47^{\star \star \star}$ \\
\hline Mean & $r_{\mathrm{s}}$ & $0.52^{\star \star *}$ & $0.60^{\star \star *}$ & $0.51^{* \star *}$ & $0.56^{\star \star \star}$ & $0.51^{* * *}$ & $0.57^{\star \star \star}$ \\
\hline Adjusted mean & & 0.63 & 0.80 & 0.65 & 0.77 & 0.65 & 0.79 \\
\hline & $95 \% \mathrm{Cl}$ & $0.39,0.79$ & $0.62,0.90$ & $0.42,0.80$ & $0.58,0.89$ & $0.42,0.81$ & $0.60,0.90$ \\
\hline
\end{tabular}

DHBA, 3,5-dihydroxybenzoic acid; DHPPA, 3-(3,5-dihydroxyphenyl)-propanoic acid; CR, creatinine

Values were significantly different: ${ }^{*} P<0.05,{ }^{\star \star} P<0.01,{ }^{\star \star *} P<0.001$.

†Spearman's rank $r_{\mathrm{s}}$ were calculated from daily intake estimated by $3 \mathrm{~d}$ weighed food records (two separate occasions, 2-3 months apart, and mean of occasions) and $24 \mathrm{~h}$ AR metabolite excretion (first occasion and mean of two occasions). Observed $r_{\mathrm{s}}$ between mean daily intake and mean CR-adjusted AR metabolite concentration in morning urine were adjusted for intra-individual variation in the intake variables, $n 66$.

$\ddagger$ No correlations were found between CR-adjusted AR metabolite concentration in morning urine and mean intake of WG oats, WG barley or WG rice.

$\S$ Spearman's rank $r_{\mathrm{s}}$ between mean daily intake and $24 \mathrm{~h}$ urinary AR metabolite excretion were adjusted for within-person variation in intake variables

Adjusted $r_{\mathrm{s}}$ are presented with their $95 \% \mathrm{Cl}$

The intake of macronutrients, WG and AR was similar to that in the previous study performed on the same volunteers, although fewer participants were included in the present study $^{(24)}$. The amounts of AR metabolites excreted in the present study were similar to those for Finnish women with comparable cereal fibre intake ${ }^{(17,18)}$. The $24 \mathrm{~h}$ excretion of DHPPA in the present study was $>3$ times the $12 \mathrm{~h}$ DHPPA excretion among North American men and women ${ }^{(19)}$. The higher excretion is most probably attributable to differences in diet, especially rye intake, where only a fraction of the American participants reported intake of WG rye, whereas rye was the most common cereal consumed as WG in the present study ${ }^{(17)}$.

The reproducibility of urinary AR metabolites over 2-3 months was similar to the intake of WG and cereal fibre in the present study and comparable to the reproducibility of fasting plasma AR concentrations in the same participants ${ }^{(24)}$ and in German participants over 4 months ${ }^{(5)}$. The reproducibility was higher for $24 \mathrm{~h}$ excretion of AR metabolites than for morning urine concentration, as the latter covers a shorter collection interval. However, $24 \mathrm{~h}$ excretion of AR metabolites correlated well with AR metabolite concentration in morning urine of the same day, with equal or higher correlations than that reported for urinary $\mathrm{Na}, \mathrm{K}$ and polyphenols ${ }^{(31,32)}$. The present study showed that urinary AR metabolite excretion is comparable to fasting plasma AR concentration as a medium-term (2-3 months) biomarker. Although true elimination half-lives of AR metabolites have yet to be determined, studies on plasma and urine show that AR metabolites disappear from plasma and appear in urine rather quickly after the absorption of dietary $\mathrm{AR}^{(9,15,16)}$. This, in combination with modestly stable intakes of foods containing $\mathrm{AR}$, is reflected in the observed medium-term reproducibility of urinary AR metabolites.

Excretion of AR metabolites is well correlated to intake of AR. Rye contains high amounts of $\mathrm{AR}^{(26)}$ and was the main source of WG and cereal fibre in the present study. As a result of this, $\mathrm{AR}$ metabolite excretion correlated strongly with the intake of cereal fibre, total WG, and especially WG rye. The absence of correlations between WG wheat intake and urinary AR metabolites is probably a result of the low and unstable intake of WG wheat and high intake of WG 
rye in this population. It is possible that urinary excretion of AR metabolites could reflect WG wheat intake more successfully in populations where consumption of rye is smaller and WG wheat intake is more stable ${ }^{(19)}$. Due to the absence of AR in WG oats, maize and rice and their low concentrations in WG barley, these cereals make negligible contributions to AR consumption and, as expected, they did not correlate to urinary excretion of AR metabolites. The correlations between intakes and AR metabolite excretion were stronger in $24 \mathrm{~h}$ collections than in morning urine, due to lower reproducibility of the latter. The advantages with morning urine samples compared with $24 \mathrm{~h}$ collections (e.g. greater compliance) need to be considered in the light of the disadvantages (e.g. lower reproducibility) when designing new studies. By estimating the variance components in a reproducibility study, the observed correlations can be adjusted for intra-individual variation in intake and thereby allow the estimation of correlations between 'true' intake and biomarker measurement ${ }^{(33)}$. In most cases, it is preferable to estimate variance components from few repeated measurements in a large number of participants, rather than from numerous repeated measurements in a small group of participants ${ }^{(33)}$. Adjustments of intra-individual variations in intake substantially improved correlations between intake and excretion, although $95 \%$ CI of correlation coefficients also increased due to few participants and moderate intake reproducibility. In this study, the correlations between intake and $24 \mathrm{~h}$ excretion were comparably improved when adjusting for either variations in intake or excretion, due to their similarities in reproducibility (Tables 1 and 2). Differently, morning urine concentrations were less stable than intake, which resulted in stronger corrected correlations when adjusting for intra-individual variation in urine concentration compared to intake variation. Intake during the separate occasions was more strongly correlated to excretion on the corresponding occasion than to the opposite occasion, since excretion of AR metabolites primarily reflects short-term intake and intakes were only moderately stable over the two occasions. In this study, intakes of WG rye and wheat, WG rye and cereal fibre were reflected by urinary AR metabolites measured 2-3 months before and after intake

Table 4. Observed and adjusted Spearman's rank $r_{\mathrm{s}}$ between creatinine (CR)-adjusted alkylresorcinols (AR) metabolite concentration in morning urine and intake of total whole grain (WG), WG rye, WG wheat and cereal fibreł‡

(Mean values with their $95 \%$ confidence intervals)

\begin{tabular}{|c|c|c|c|c|c|c|c|}
\hline & & \multicolumn{2}{|c|}{ DHBA } & \multicolumn{2}{|c|}{ DHPPA } & \multicolumn{2}{|c|}{ DHBA + DHPPA } \\
\hline & & First occasion & Mean & First occasion & Mean & First occasion & Mean \\
\hline \multicolumn{8}{|l|}{ Total WG } \\
\hline First occasion & $r_{\mathrm{s}}$ & $0.46^{\star \star *}$ & $0.34^{\star *}$ & $0.47^{\star \star \star}$ & $0.36^{\star \star}$ & $0.46^{\star \star \star}$ & $0.38^{\star \star}$ \\
\hline Second occasion & $r_{\mathrm{s}}$ & 0.23 & $0.34^{\star *}$ & 0.21 & $0.28^{*}$ & 0.21 & $0.32^{\star *}$ \\
\hline Mean & $r_{\mathrm{s}}$ & $0.38^{\star \star}$ & $0.37^{* *}$ & $0.37^{\star *}$ & $0.35^{\star *}$ & $0.37^{\star *}$ & $0.39^{* *}$ \\
\hline Adjusted mean§ & $r_{\mathrm{s}}$ & 0.49 & 0.50 & 0.50 & 0.50 & 0.49 & 0.53 \\
\hline & $95 \% \mathrm{Cl}$ & $0.23,0.69$ & $0.25,0.59$ & $0.24,0.69$ & $0.24,0.69$ & $0.59,0.69$ & $0.27,0.71$ \\
\hline \multicolumn{8}{|l|}{ WG rye and wheat } \\
\hline First occasion & $r_{\mathrm{s}}$ & $0.53^{\star \star \star}$ & $0.41^{\star \star \star}$ & $0.53^{\star \star \star}$ & $0.42^{* \star *}$ & $0.53^{\star \star \star}$ & $0.44^{\star * \star}$ \\
\hline Second occasion & $r_{\mathrm{s}}$ & $0.32^{\star \star}$ & $0.49^{\star * *}$ & $0.25^{\star}$ & $0.40^{\star \star \star}$ & $0.28^{*}$ & $0.45^{\star \star \star}$ \\
\hline Mean & $r_{\mathrm{s}}$ & $0.49^{\star \star \star}$ & $0.53^{\star \star \star}$ & $0.45^{\star \star \star}$ & $0.47^{\star \star \star}$ & $0.47^{\star \star \star}$ & $0.52^{\star \star \star}$ \\
\hline Adjusted mean & $r_{\mathrm{s}}$ & 0.61 & 0.65 & 0.58 & 0.62 & 0.59 & 0.66 \\
\hline & $95 \% \mathrm{Cl}$ & $0.37,0.78$ & $0.42,0.81$ & $0.33,0.76$ & $0.38,0.78$ & $0.35,0.77$ & $0.43,0.81$ \\
\hline \multicolumn{8}{|l|}{ WG rye } \\
\hline First occasion & $r_{\mathrm{s}}$ & $0.53^{\star \star *}$ & $0.58^{\star \star \star}$ & $0.59^{\star \star \star}$ & $0.60^{\star * *}$ & $0.58^{\star \star \star}$ & $0.62^{\star \star \star}$ \\
\hline Second occasion & $r_{\mathrm{s}}$ & $0.32^{\star \star}$ & $0.48^{\star \star \star}$ & $0.35^{\star \star}$ & $0.49^{\star \star \star}$ & $0.34^{\star \star}$ & $0.51^{\star * \star}$ \\
\hline Mean & $r_{\mathrm{s}}$ & $0.47^{\star \star \star}$ & $0.59^{\star \star \star}$ & $0.52^{\star \star \star}$ & $0.60^{\star \star \star}$ & $0.51^{\star \star \star}$ & $0.63^{\star \star \star}$ \\
\hline Adjusted mean & $r_{\mathrm{s}}$ & 0.56 & 0.69 & 0.66 & 0.75 & 0.64 & 0.75 \\
\hline & $95 \% \mathrm{Cl}$ & $0.33,0.74$ & $0.49,0.83$ & $0.44,0.80$ & $0.56,0.86$ & $0.42,0,79$ & $0.56,0.87$ \\
\hline \multicolumn{8}{|l|}{ WG wheat } \\
\hline First occasion & $r_{\mathrm{s}}$ & 0.19 & -0.01 & 0.12 & -0.06 & 0.13 & -0.03 \\
\hline Second occasion & $r_{\mathrm{s}}$ & 0.03 & 0.03 & -0.12 & -0.03 & -0.10 & 0.00 \\
\hline Mean & $r_{\mathrm{s}}$ & 0.13 & 0.01 & 0.03 & -0.05 & 0.05 & -0.02 \\
\hline Adjusted mean & $r_{\mathrm{s}}$ & 0.15 & 0.03 & -0.01 & -0.06 & 0.02 & -0.01 \\
\hline & $95 \% \mathrm{Cl}$ & $-0.14,0.42$ & $-0.25,0.31$ & $-0.29,0.27$ & $-0.34,0.23$ & $-0.26,0.30$ & $-0.29,0.27$ \\
\hline \multicolumn{8}{|l|}{ Cereal fiber } \\
\hline First occasion & $r_{\mathrm{s}}$ & $0.53^{\star \star \star}$ & $0.38^{* *}$ & $0.55^{\star \star \star}$ & $0.41^{\star \star \star}$ & $0.54^{\star \star \star}$ & $0.42^{\star \star \star}$ \\
\hline Second occasion & $r_{\mathrm{s}}$ & $0.27^{*}$ & $0.35^{\star *}$ & $0 \cdot 30^{*}$ & $0.35^{\star \star}$ & $0.29^{\star}$ & $0.38^{\star \star}$ \\
\hline Mean & $r_{\mathrm{s}}$ & $0.46^{\star \star \star}$ & $0.39^{\star \star}$ & $0.48^{\star \star \star}$ & $0.41^{\star \star \star}$ & $0.47^{\star \star \star}$ & $0.44^{\star \star \star}$ \\
\hline \multirow[t]{2}{*}{ Adjusted mean } & & 0.57 & 0.56 & 0.63 & 0.60 & 0.61 & 0.62 \\
\hline & $95 \% \mathrm{Cl}$ & $0.32,0.75$ & $0.31,0.74$ & $0.39,0.79$ & $0.36,0.77$ & $0.37,0.78$ & $0.39,0.78$ \\
\hline
\end{tabular}

DHBA, 3,5-dihydroxybenzoic acid; DHPPA, 3-(3,5-dihydroxyphenyl)-propanoic acid.

Values were significantly different: ${ }^{\star} P<0.05,{ }^{\star \star} P<0.01,{ }^{\star \star \star} P<0.001$.

† Spearman's rank $r_{\mathrm{s}}$ were calculated from daily intake estimated by $3 \mathrm{~d}$ weighed food records (two separate occasions, 2-3 months apart, and mean of occasions) and CR-adjusted AR metabolite concentration in morning urine (first occasion and mean of occasions). Observed $r_{\mathrm{s}}$ between mean daily intake and mean CR-adjusted AR metabolite concentration in morning urine were adjusted for intra-individual variation in the intake variables, $n 66$.

$\ddagger$ No correlations were found between CR-adjusted AR metabolite concentration in morning urine and mean intake of WG oats, WG barley or WG rice.

$\S$ Spearman's rank $r_{\mathrm{s}}$ between mean daily intake and CR-adjusted AR metabolite concentration were adjusted for within-person variation in intake variables. Adjusted $r_{\mathrm{s}}$ are presented with their $95 \% \mathrm{Cl}$. 


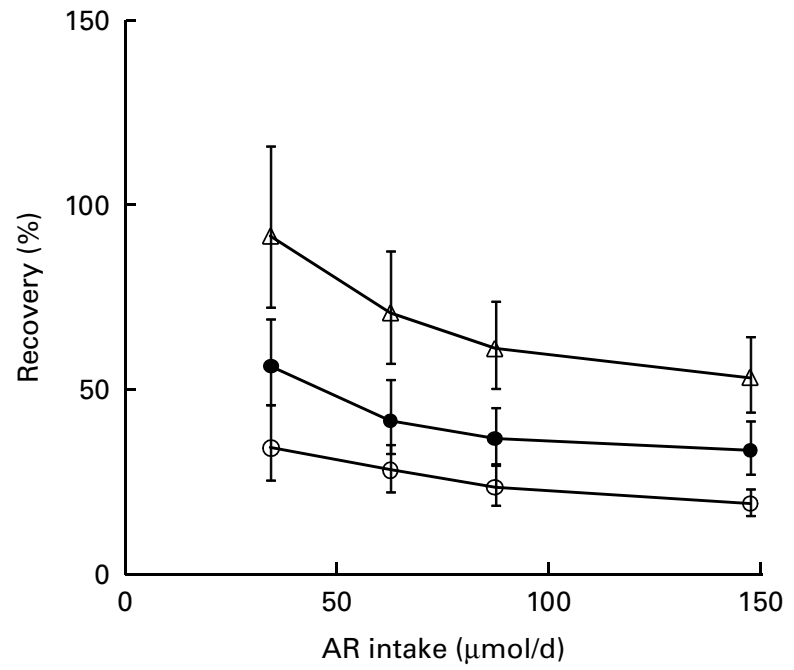

Fig. 1. Recovery of daily alkylresorcinol (AR) intake as $24 \mathrm{~h}$ urinary excretion of AR metabolites: 3,5-dihydroxybenzoic acid (DHBA, $\triangle, P$ for trend $<0.001)$ and 3-(3,5-dihydroxyphenyl)-propanoic acid (DHPPA, - - $P$ for trend $<0.01)$, and their total $(-, P$ for trend $<0.001)$. Participants who consumed $\mathrm{AR}$ and provided $24 \mathrm{~h}$ urine collections plus acceptable $3 \mathrm{~d}$ weighed food records on both occasions were included ( $n$ 62) and divided into quartiles based on AR intake. The quartile recoveries (geometric means) were plotted against median intake per quartile. Error bars represent $95 \%$ $\mathrm{Cl}$ of mean recoveries.

measurement. However, if urinary AR metabolites in single samples are to be used to reflect long-term intakes of WG or fibre, variance components of preferably both intake and excretion should be reliably addressed in the study population in order to allow adjustment for intra-individual variations.

The proportion of ingested AR recovered as AR metabolites in urine decreased with increasing AR intake, a phenomenon that has also been observed in other studies ${ }^{(3,17)}$. Elimination of AR metabolites is thought to be not exclusively through urinary excretion, but also to some extent through excretion in bile ${ }^{(3)}$. It has been suggested that absorption of $\mathrm{AR}$ and/or the elimination of AR or their metabolites is dependent on the dose ingested, where higher amounts of ingested AR result in lower proportions of ingested AR recovered as urinary metabolites ${ }^{(3,16,17)}$. A dose-dependent shift in elimination would attenuate correlations between AR intake and urinary AR metabolites. In addition, inter-individual variations in AR elimination attributable to sources other than the amount ingested, such as polymorphism of cytochrome P450 enzymes influencing $\alpha$ - and $\gamma$-tocopherol metabolism ${ }^{(34)}$, may further weaken the correlations. Finally, alternative precursors to DHBA and DHPPA may exist, as low concentrations of both compounds have been detected in urine from individuals with no reported AR intake ${ }^{(13)}$. It is possible that DHBA and DHPPA deriving from sources other than AR might contribute to an over-estimation of recovery, especially in individuals with low AR intake. Further studies on the elimination of $\mathrm{AR}$ and their metabolites are needed to explain the apparent dose-dependent effect on recovery of ingested AR.

In conclusion, $24 \mathrm{~h}$ excretion and CR-adjusted morning urine concentration of AR metabolites correlated well. Daily urinary excretion of AR metabolites showed moderate medium-term reproducibility, comparable to fasting plasma
AR concentration and WG intake. Medium-term reproducibility of CR-adjusted AR metabolite concentration in morning urine was lower than that observed for $24 \mathrm{~h}$ excretion. In this study, urinary AR metabolites appeared to be comparable to fasting plasma $\mathrm{AR}$ as medium-term biomarkers. In this population, where consumption of rye and wheat was high ${ }^{(24)}$, urinary AR metabolites showed good relative validity as biomarkers for WG intake and correlated well with the medium-term intake of WG, fibre and AR after adjustment for intra-individual variation in intake. Our findings support the idea of urinary AR metabolites as short/medium-term biomarkers of intake of WG and cereal fibre in populations where rye is a major contributor of these dietary components. If single urine samples are to be used to reflect intake over a longer time period, variance components of intake and excretion need to be adequately assessed in the study population to allow adjustments for intra-individual variation in intake and in biomarker measurements. Further studies are needed to address the apparent dose-dependent elimination of $\mathrm{AR}$, as this might interfere with the performance of $\mathrm{AR}$ metabolites as biomarkers.

\section{Acknowledgements}

The authors thank Ina Granlund, registered dietitian, for her skilled assistance with the nutrient calculations. R. L. and A. A. designed the study; M. M. and R. L. conducted the clinical part of the study; A. A. supervised the nutrient calculations; M. M. performed the analysis of urinary AR metabolites under the supervision of A. K.-E., P. A. and R. L.; M. M. performed the statistical analysis and wrote the manuscript under the supervision of R. L.; M. M. has the primary responsibility for the final content; A. K.-E. and P. A. initiated the AR biomarker research; all authors read and approved the final manuscript. The present study was supported by grants from FORMAS: The Swedish Research Council for Environment, Agricultural Sciences and Spatial Planning (A. K.-E., M. M.); Nordic Centre of Excellence Programme on Food, Nutrition and Health: HELGA Nordic Health-Whole Grain Food (R. L., P. A.); The Faculty Board for Medicine and Pharmacy at Uppsala University (A. A.); The Royal Swedish Academy of Agriculture and Forestry (A. A., R. L.); Swedish Nutrition Foundation (M. M., R. L.). There were no conflicts of interest.

\section{References}

1. Linko-Parvinen AM, Landberg R, Tikkarren MJ, et al. (2007) Alkylresorcinols from whole-grain wheat and rye are transported in human plasma lipoproteins. J Nutr 137, 1137-1142.

2. Landberg R, Kamal-Eldin A, Andersson A, et al. (2008) Alkylresorcinols as biomarkers of whole-grain wheat and rye intake: plasma concentration and intake estimated from dietary records. Am J Clin Nutr $\mathbf{8 7}, 832-838$.

3. Landberg R, Aman P, Friberg LE, et al. (2009) Dose response of whole-grain biomarkers: alkylresorcinols in human plasma and their metabolites in urine in relation to intake. Am J Clin Nutr 89, 290-296.

4. Landberg R, Kamal-Eldin A, Andersson SO, et al. (2009) Reproducibility of plasma alkylresorcinols during a 6-week 
rye intervention study in men with prostate cancer. $J$ Nutr 139, 975-980.

5. Montonen J, Landberg R, Kamal-Eldin A, et al. (2010) Reliability of fasting plasma alkylresorcinol concentrations measured 4 months apart. Eur J Clin Nutr 64, 698-703.

6. Landberg R, Kamal-Eldin A, Salmenkallio-Marttila M, et al. (2008) Localization of alkylresorcinols in wheat, rye and barley kernels. J Cereal Sci 48, 401-406.

7. Ross AB \& Kochhar S (2009) Rapid and sensitive analysis of alkylresorcinols from cereal grains and products using HPLCCoularray-based electrochemical detection. J Agric Food Chem 57, 5187-5193.

8. Andersson AAM, Lampi A-M, Nyström L, et al. (2008) Phytochemical and dietary fiber components in barley varieties in the HEALTHGRAIN Diversity Screen. J Agric Food Chem 56, 9767-9776.

9. Landberg R, Linko AM, Kamal-Eldin A, et al. (2006) Human plasma kinetics and relative bioavailability of alkylresorcinols after intake of rye bran. J Nutr 136, 2760-2765.

10. Sontag TJ \& Parker RS (2002) Cytochrome P450 $\omega$-hydroxylase pathway of tocopherol catabolism. J Biol Chem 277, $25290-25296$.

11. Ross AB, Aman P \& Kamal-Eldin A (2004) Identification of cereal alkylresorcinol metabolites in human urine - potential biomarkers of wholegrain wheat and rye intake. J Chromatogr B 809, 125-130.

12. Koskela A, Samaletdin A, Aubertin-Leheudre MN, et al. (2008) Quantification of alkylresorcinol metabolites in plasma by high-performance liquid chromatography with coulometric electrode array detection. J Agric Food Chem 56, 7678-7681.

13. Koskela A, Linko-Parvinen AM, Hiisivuori P, et al. (2007) Quantification of alkylresorcinol metabolites in urine by HPLC with coulometric electrode array detection. Clin Chem 53, 1380-1383.

14. Marklund M, Landberg R, Aman P, et al. (2010) Determination of alkylresorcinol metabolites in human urine by gas chromatography-mass spectrometry. $J$ Chromatogr $B$ 878, 888-894.

15. Soderholm PP, Koskela AH, Lundin JE, et al. (2009) Plasma pharmacokinetics of alkylresorcinol metabolites: new candidate biomarkers for whole-grain rye and wheat intake. $A m J$ Clin Nutr 90, 1167-1171.

16. Söderholm PP, Lundin JE, Koskela AH, et al. (2011) Pharmacokinetics of alkylresorcinol metabolites in human urine. BrJ Nutr 106, 1040-1044.

17. Aubertin-Leheudre M, Koskela A, Samaletdin A, et al. (2010) Responsiveness of urinary and plasma alkylresorcinol metabolites to rye intake in finnish women. Cancers 2, 513-522.

18. Aubertin-Leheudre M, Koskela A, Marjamaa A, et al. (2008) Plasma alkylresorcinols and urinary alkylresorcinol metabolites as biomarkers of cereal fiber intake in finnish women. Cancer Epidemiol Biomarkers Prev 17, 2244-2248.

19. Guyman LA, Adlercreutz H, Koskela A, et al. (2008) Urinary 3-(3,5-dihydroxyphenyl)-1-propanoic acid, an alkylresorcinol metabolite, is a potential biomarker of whole-grain intake in a US population. J Nutr 138, 1957-1962.

20. Aubertin-Leheudre M, Koskela A, Samaletdin A, et al. (2010) Plasma and urinary alkylresorcinol metabolites as potential biomarkers of breast cancer risk in Finnish women: a pilot study. Nutr Cancer 62, 759-764.

21. Marklund M, Landberg R, Åman P, et al. (2011) Comparison of gas chromatography-mass spectrometry and high-performance liquid chromatography with coulometric electrode array detection for determination of alkylresorcinol metabolites in human urine. J Chromatogr B 879, 647-651.

22. Rivera-Núñez Z, Meliker JR, Linder AM, et al. (2010) Reliability of spot urine samples in assessing arsenic exposure. Int J Hyg Environ Health 213, 259-264.

23. Ohira S, Kirk AB, Dyke JV, et al. (2008) Creatinine adjustment of spot urine samples and $24 \mathrm{~h}$ excretion of iodine, selenium, perchlorate, and thiocyanate. Environ Sci Technol 42, 9419-9423.

24. Andersson A, Marklund M, Diana M, et al. (2011) Plasma alkylresorcinol concentrations correlate with whole grain wheat and rye intake and show moderate reproducibility over a 2- to 3-month period in free-living Swedish adults. J Nutr 141, 1712-1718.

25. AACC International (1999) Definition of whole grain. http:// www.aaccnet.org/definitions/wholegrain.asp (accessed 8 August 2011).

26. Ross AB, Shepherd MJ, Schupphaus M, et al. (2003) Alkylresorcinols in cereals and cereal products. J Agric Food Chem 51, 4111-4118.

27. Rosner B (2009) Attenuation point estimate and coefficients for measurement - error corrected rank https://sites.google. $\mathrm{com} / \mathrm{a} /$ channing.harvard.edu/bernardrosner/channing/interval-estimation-for-rank-correlation-coefficients/attenuationpoint-estimate-and-coefficients-for-measurement-error-corrected-rank (accessed 30 November 2011).

28. Rosner B \& Glynn RJ (2007) Interval estimation for rank correlation coefficients based on the probit transformation with extension to measurement error correction of correlated ranked data. Stat Med 26, 633-646.

29. Garde AH, Hansen ÅM, Kristiansen J, et al. (2004) Comparison of uncertainties related to standardization of urine samples with volume and creatinine concentration. Ann Occup Hyg 48, 171-179.

30. Kaaks RJ (1997) Biochemical markers as additional measurements in studies of the accuracy of dietary questionnaire measurements: conceptual issues. Am J Clin Nutr 65 , 1232S-1239S

31. Mennen LI, Sapinho D, Ito H, et al. (2008) Urinary excretion of 13 dietary flavonoids and phenolic acids in free-living healthy subjects - variability and possible use as biomarkers of polyphenol intake. Eur J Clin Nutr 62, 519-525.

32. Ilich JZ, Blanusa M, Orlic ZC, et al. (2009) Comparison of calcium, magnesium, sodium, potassium, zinc, and creatinine concentration in 24-h and spot urine samples in women. Clin Chem Lab Med 47, 216-221.

33. Rosner B \& Willett WC (1988) Interval estimates for correlation coefficients corrected for within-person variation: implications for study design and hypothesis testing. $\mathrm{Am} \mathrm{J}$ Epidemiol 127, 377-386.

34. Bardowell SA, Stec DE \& Parker RS (2010) Common variants of cytochrome P450 4F2 exhibit altered vitamin E-\{omega\}hydroxylase specific activity. J Nutr 140, 1901-1906. 